system, rather than to the celebrated value of $R(\ln 2-1 / 2 \ln (3 / 2))$, which corresponds to the Pauling entropy observed ${ }^{2}$ in bulk $\mathrm{Dy}_{2} \mathrm{Ti}_{2} \mathrm{O}_{7}$ (and famously measured by Giauque and Stout in water ice in the $\left.1930 s^{9}\right)$. This observation prompts Bovo and colleagues to argue that the third law of thermodynamics, violated in bulk spin ice materials, is restored in thin films ${ }^{6}$. They suggest the most likely cause for this may be the strain induced by the lattice mismatch between the spin ice film and its substrate.

Whether or not strain is indeed the cause of this phenomenon remains to be proven, but there is no doubt that the fact that a new field for addressing a series of probing questions is now well and truly open. Apart from the important but perhaps more mundane issues concerning the quality of the thin films and the influence of the substrate on their properties, some of the more interesting questions the works of Bovo et al. ${ }^{6}$ and Leusink et al. ${ }^{7}$ raise are: What is the ground state of a monolayer-thick spin ice? Can the spin ice films be properly thermally equilibrated even at the lowest temperatures on a timescale of typical experiments? Are monopole dynamics different on the surface and can they be manipulated? Can any links to the growing body of work on artificial spin ice ${ }^{10}$ be made? Doubtless there will be surprises, but these issues will keep researchers busy in the field of magnetism and beyond.
Oleg Petrenko is at the Department of

Physics, University of Warwick, Coventry

CV4 7AL, UK.

e-mail:O.Petrenko@warwick.ac.uk

References

1. Harris, M. J., Bramwell, S. T., McMorrow, D. F., Zeiske, T. \& Godfrey, K. W. Phys. Rev. Lett. 79, 2554-2557 (1997).

2. Ramirez, A. P., Hayashi, A., Cava, R. J., Siddharthan, R. \& Shastry, B. S. Nature 399, 333-335 (1999).

3. Castelnovo, C., Moessner, R. \& Sondhi, S. L. Nature 451, 42-45 (2008)

4. Ryzhkin, I. A. Sov. Phys JETP 101, 481-486 (2005).

5. Giblin, S. R. et al. Nature Phys. 7, 252-258 (2011).

6. Bovo, L. et al. Nature Commun. 5, 3439 (2014).

Leusink, D. P. et al. APL Mater. 2, 032101 (2014).

8. Petrenko, V. F. \& Whitworth, R. W. Physics of Ice (Oxford Univ. Press, 1999).

9. Giauque, W. F \& Stout J. W. J. Am. Chem. Soc 58, 1144-1150 (1936)

10. Nisoli, C., Moessner, R. \& Schiffer, P. Rev. Mod. Phys. 85, 1473-1490 (2013)

\title{
STELLAR METALLURGY
}

The recent detection of a fingerprint in the polarization of the cosmic microwave background radiation, apparently left by primordial gravitational waves triggered by an inflationary epoch in the universe's expansion after the Big Bang, has been hailed as the kind of discovery that comes only once every few decades. Although such phenomena seem a very long way from the science of materials, the ongoing search for a direct detection of gravitational waves produced in violent astrophysical events certainly is not.

Candidate sources for these waves include rotating neutron stars with 'mountains' on their surface - small deviations from perfect rotational symmetry of the high-density crust, which would excite and radiate gravitational waves. The higher the mountains, the stronger the waves. How high the mountains are - and we're talking inches here - depends on the strength of the crust.

The crust's materials properties could also play a role in understanding other phenomena. For example, 'star quakes' produced by magnetic stresses that deform and ultimately crack the crust of magnetized neutron stars have been proposed as a possible explanation for gamma-ray flares seen in 2004 from SGR 1806-20, an object thought to be a neutron star in the Sagittarius constellation ${ }^{1}$.

Whereas a neutron star's deep interior is thought to be essentially a neutron fluid, the crust contains the nuclei of neutron-rich atoms immersed in an electron gas and permeated by a superfluid of free neutrons. The nuclei have been long assumed to form a body-centred cubic (bcc) lattice, and on this basis the strength of the crust has previously been estimated to be around 10 billion times that of steel ${ }^{2}$. That study modelled the ultradense fabric of neutron stars much like the crystal lattice of a metal, complete with dislocations (albeit mostly squeezed out by the intense pressure).

Now Kobyakov and Pethick extend the metallurgical picture further, and in doing so they revise our view of the material properties of the crust of a neutron star ${ }^{3}$. They argue that the interstitial neutron fluid between the nuclei acts like a second component of an alloy, and has the effect of creating an attraction between the nuclei that renders the bcc lattice unstable to a phase transition, in a manner analogous to the phase separation of an alloy through spinodal decomposition ${ }^{4}$.

Although it isn't possible from this stability analysis alone to calculate what structure the phase transition will produce, the nature of the most unstable mode suggests a

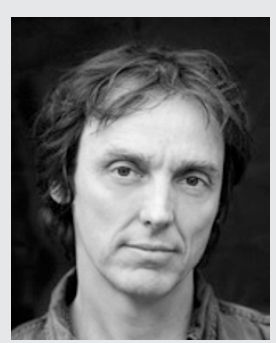

PHILIP BALL

doubling of the unit cell, which Kobyakov and Pethick interpret as perhaps leading to a phase analogous to a ferroelectric such as barium titanate (but without actual ferroelectricity). This would be likely to increase the crust's breaking strain, and also to alter the thermodynamic and transport properties relevant to star quakes and other phenomena. There are evidently, then, implications for astrophysics and gravitational-wave detection. But in the application of methods developed for metallurgy to these exotic and barely imaginable materials, there is also an illustration of the unity in condensed-matter physics.

\section{References}

1. Hurley, K. et al. Nature 434, 1098-1103 (2005)

2. Horowitz, C. J. \& Kadau, K. Phys. Rev. Lett. 102, 191102 (2009).

3. Kobyakov, D. \& Pethick, C. J. Phys. Rev. Lett. 112, 112504 (2014).

4. Cahn, J. W. Acta Metall. 10, 179-183 (1962) 